\title{
All Kinds of Alkynylpyridiniums: Elusive Salts Now Plentiful
}
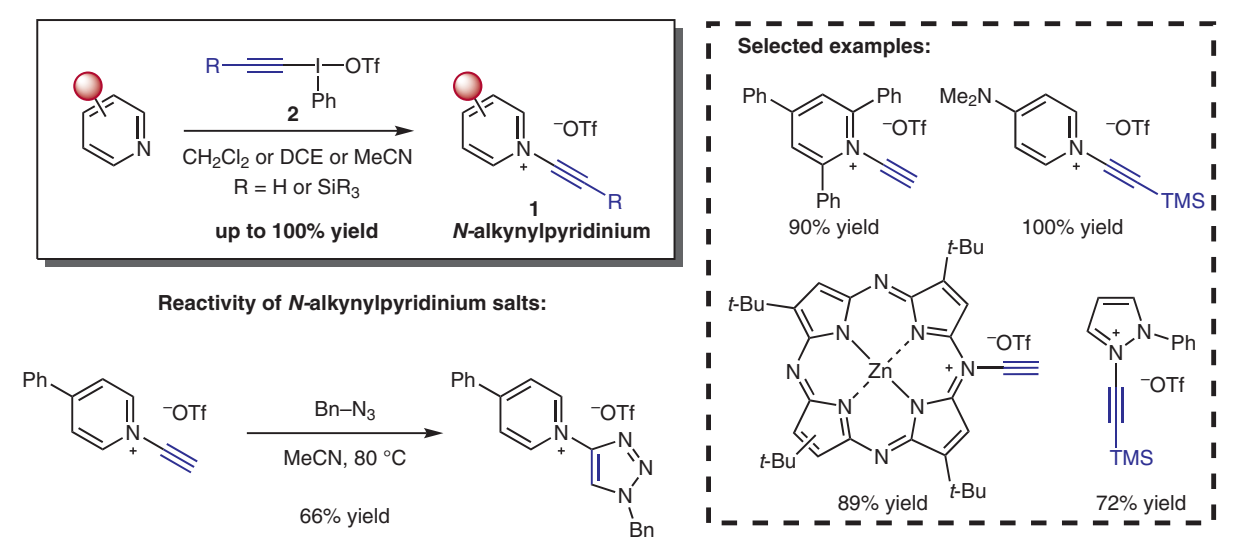


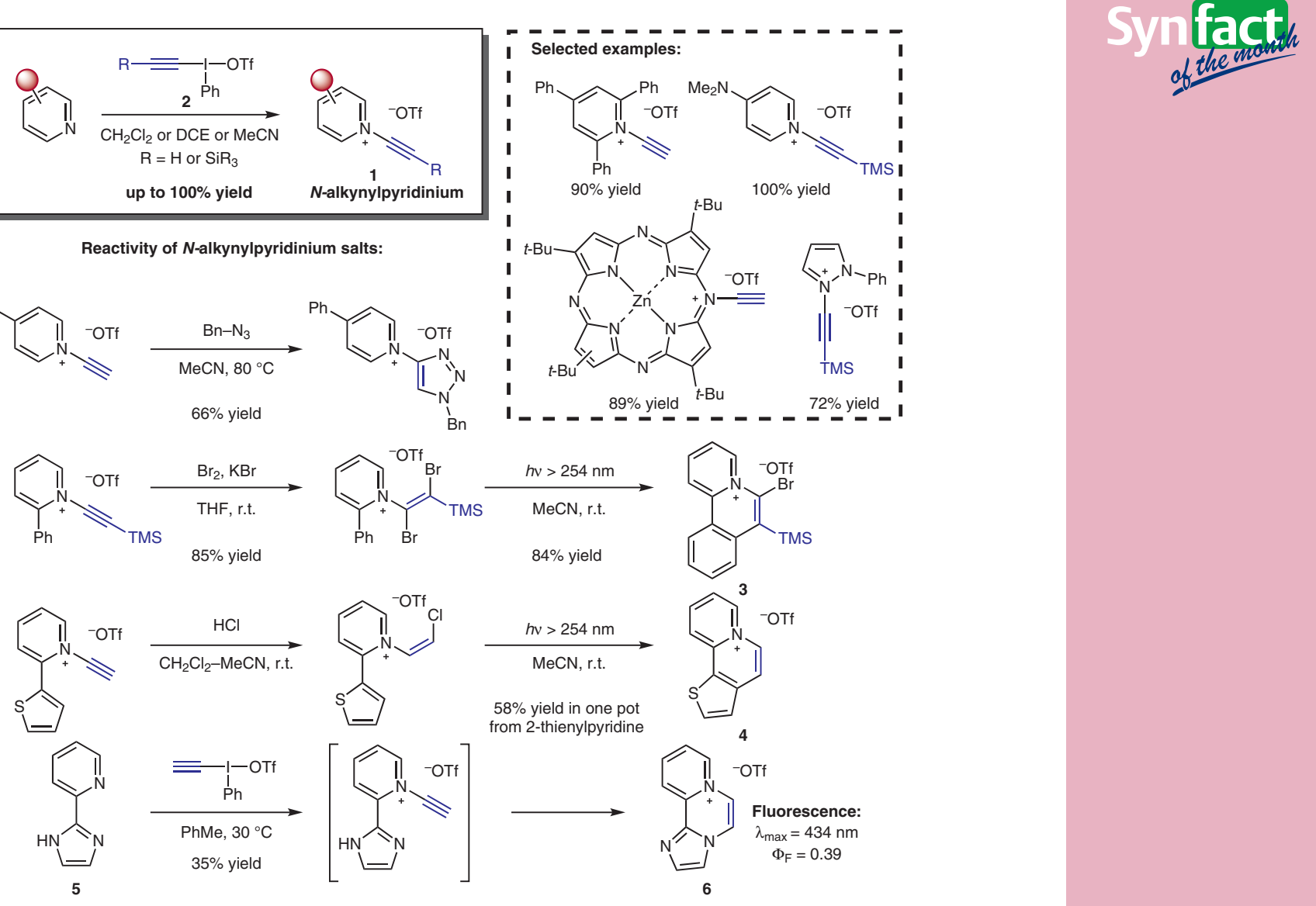

Significance: N-Alkynylpyridinium salts (e.g., 1) have eluded isolation in previous attempts to synthesize these electrophilic molecules. Herein, the researchers disclose that alkynyl iodanes $\mathbf{2}$ are highly effective reagents for the preparation of a wide range of $N$-alkynylpyridinium triflates directly from pyridines. This method enabled the first full characterization of an $N$-alkynylpyridinium salt and the exploration of further transformations, including the synthesis of 'nitrogen-doped' polycyclic aromatic compounds with promising electronic properties for materials applications.

SYNFACTS Contributors: Timothy M. Swager, Nathan A. Romero Synfacts 2018, 14(05), 0471 Published online: 17.04.2018 DOI: 10.1055/s-0037-1609689; Reg-No.: S02518SF

\section{Key words}

\section{pyridinium}

hypervalent iodine

photocyclization
Comment: Excellent yields are reported for the alkynylation of variously substituted pyridines as well as other aza-heterocycles. The $N$-alkylpyridinium triflates are stable under ambient conditions for at least six months, but the alkyne unit can be transformed in dipolar cycloaddition, halogenation, and hydrofunctionalization reactions. Halogenated products bearing a 2-aryl substituent were further subjected to photocyclizationdehydrohalogenation to generate quinolizinium salts (e.g., 3 and 4), and fluorescent compounds such as $\mathbf{6}$ were formed directly in the alkynylation of 2-imidazolylpyridines $\mathbf{5}$. 